\title{
Faunal diversity of the benthic amphipods (Crustacea) of the Magellan region as compared to the Antarctic (preliminary results)*
}

\author{
CLAUDE DE BROYER ${ }^{1}$ and MARTIN RAUSCHERT ${ }^{2}$ \\ ${ }^{1}$ Institut royal des Sciences naturelles de Belgique, rue Vautier 29, B-1000 Bruxelles, Belgium. Email: \\ debroyer@kbinirsnb.be \\ ${ }^{2}$ Alfred-Wegener-Institut für Polar- und Meeresforschung, Forschungsstelle Postdam, c/o Museum für Naturkunde, \\ Invalidenstraße 43, D-10115 Berlin, Germany. Email: martin.rauschert@rz.hu-berlin.de
}

\begin{abstract}
SUMMARY: To investigate the marine benthic ecosystems of the Magellan region and to compare them with the better known Antarctic systems, three campaigns were recently carried out in this area: the Joint Magellan "Victor Hensen" Campaign 1994, the "Polarstern" ANT XIII/4 cruise 1996, and the "Vidal Gormaz" CIMAR FIORDO II cruise 1996. Numerous and diverse zoobenthos samples were collected mostly with an Agassiz trawl and with a small dredge, an epibenthic sledge, with baited traps or by diving. All gears together gathered more than 132,000 specimens of gammaridean and caprellidean amphipods. 137 species of gammaridean amphipods have been identified from the material to date. About $20 \%$ of these species appear to be new for science. This taxonomic work takes place in the framework of a general revision of the Southern Ocean amphipod fauna undertaken by the"Antarctic Amphipodologists Network". A complete list of the benthic species of gammaridean and caprellidean amphipods is presented, including the zoogeographical distribution and the new records. The new abundant material collected, still under study, will allow a comparison of faunal diversity, zoogeographical and ecological traits of the Magellan benthic amphipod taxocoenoses with those of the West and East Antarctic benthos.
\end{abstract}

Key words: Biodiversity, Amphipoda, benthos, Magellan region, zoogeography.

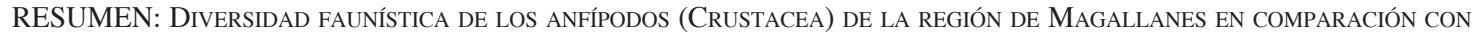
LA DE LA ANTÁRTIDA (RESULTADOS PRELIMINARES). - En el área de Magallanes se han Ilevado a cabo recientemente tres campañas de investigación: la campaña con B/I "Victor Hensen" en 1994, y las campañas "Polarstern" ANT XIII/4 y CIMAR FIORDO II con el B/O "Vidal Gormáz" en 1996. En las tres campañas se recoltaron numerosas y muy variadas muestras de organismos bentónicos. Las muestras se recogieron principalmente con la red Agassiz y mediante buceo. Los anfípodos se recolectaron también mediante una pequeña rastra y con trampas con cebo. En el conjunto de todas las muestras se recolectaron más de 132.000 especímenes de anfípodos gammáridos y caprélidos. La mayor parte de estos ejemplares se ha identificado, habiéndose obtenido unas 137 especies de las que un $20 \%$ son especies nuevas para la ciencia. El trabajo taxonómico efectuado se ha desarrollado en el marco del programa de revisión de la fauna de anfípodos del Oceáno Austral que lleva a cabo la "Antarctic Amphipodologists Network". El abundante material nuevo recolectado, en parte aún por estudiar, permitirá reevaluar las afinidades zoogeográficas, el origen y la dispersión de la fauna de anfípodos bentónicos de la región de Magallanes en relación con la fauna antártica del grupo.

Palabras clave: Biodiversidad, Amphipoda, bentos, región de Magallanes, zoogeografía. 


\section{INTRODUCTION}

The marine benthic fauna and flora of the Magellan area as well as the structure and function of its benthic ecosystems remain poorly known in comparison with the more quickly developing knowledge of the contiguous Antarctic ocean. Three different campaigns were recently carried out to investigate the Magellan area: the Joint Magellan "Victor Hensen" Campaign 1994 (Arntz and Gorny, 1996), the "Polarstern" ANT XIII/4 cruise 1996 (Fahrbach and Gerdes, 1997; Arntz et al., 1997) and the "Vidal Gormaz" CIMAR FIORDO II cruise 1996 (Mutschke et al., 1995). Numerous and diverse zoobenthos samples were collected in the channels between Puerto Montt and Puerto Natales ("Vidal Gormaz"), in the Magellan Straits, in the Magdalena, Cockburn, Brecknock, Ballenero and Beagle Channels, in the region off the eastern entrance of the Beagle Channel down to Cape Horn ("Victor Hensen") and on the northern slope of the Drake Passage ("Polarstern").

The objectives of the amphipod study conducted in these three cruises were to compare faunal diversity, biogeographical traits and ecological roles of the benthic amphipod taxocoenoses of the Magellan region with the West and East Antarctic benthos (De Broyer and Rauschert, 1996). This paper presents some first results on the composition and affinities of the benthic amphipod fauna of the Magellan region.

\section{MATERIAL AND METHODS}

Most of the zoobenthos samples were collected with an Agassiz trawl (frame $150 \times 50 \mathrm{~cm}$, "Polarstern": 300 X $100 \mathrm{~cm}$; mesh size in codend: $10 \mathrm{~mm}$ ) and some by diving with hand nets. The bulk of the amphipod material studied here was sampled using a small dredge (frame of $48 \times 18 \mathrm{~cm}$, mesh size: $1.0 \mathrm{~mm}$; "Victor Hensen" cruise: 0.3 $\mathrm{mm}$ ). In addition, baited traps and a suprabenthic sledge (Brandt et al., 1996) were used during the "Victor Hensen" and the "Polarstern" campaigns.

Sampling equipment (including the suprabenthic sledge) together provided more than 132,000 specimens of gammaridean and caprellidean amphipods. Identification of the material is still under way. The systematic arrangement used here refers to De Broyer and Jazdzewski $(1993 ; 1996)$ as well as the zoogeographical scheme which is based on Hedgpeth (1969).

\section{PRELIMINARY RESULTS}

\section{Species richness and taxonomic diversity}

According to De Broyer and Jazdzewski (1993, 1996), the gammaridean amphipod fauna of the Magellan area comprises 174 benthic and pelagic species belonging to 104 genera and 36 families. The part of the new benthic material identified to date from all three cruises consists of 137 species (Table 1). About twenty percent (28 species) of all these species appear to be new for science (including 2 spp already described by Rauschert, 1996, 1998). The new species belong to the families Cyproideidae, Eusiridae s.l., Gammaridae s.l., Liljeborgiidae, Lysianassidae s.l., Stegocephalidae and Stenothoidae. The new additions brought the Magellan gammaridean amphipod fauna to at least 206 spp (including 2 spp described by Alonso de Pina, 1997), 113 genera and 42 families (Table 2). A list of the Magellan caprellidean amphipods is given in Table 1 but the new material is still under study.

The material from the "Vidal Gormaz" CIMAR FIORDO II cruise 1996, which is nearly completely identified, comprises 68 species, which seems relatively few in comparison with the number of species from the two other cruises. However, the channels between Puerto Montt and Puerto Natales - where this material was collected - lie mostly under the influence of large glaciers and high sedimentation rates have a negative impact on zoobenthos diversity. The amphipod fauna, and the benthic fauna in general, immediately in front of the glaciers is poor in species as well as in individuals. The bulk of the amphipod fauna that was collected from the front of glaciers is formed of species from the burrower families Oedicerotidae and Phoxocephalidae. On the other hand, the amphipod samples collected by "Vidal Gormaz" from open sea areas appear richer in species than the Beagle Channel and the Magellan Strait fauna. However, the species composition shows a slightly different picture. Detailed analysis and comparison of the faunal diversity within the different Magellan areas (and with the Antarctic sub-regions) await the completion of processing of the whole material of the three expeditions. Present results nevertheless allow a preliminary comparison of the species occurrence in the East and West Antarctic and the Magellan sub-regions (Table 1). The presence in the Magellan sub-region of at least 11 genera (Eusiroides, Lepidepecreoides, Melphidippa, Mesoproboloides, Oradarea, Platyischno- 
TABLE 1. - List of benthic gammaridean and caprellidean amphipod species known from the Magellan sub-region (including the provisional list of new records) and their occurrence in the East and West Antarctic sub-regions, the Subantarctic Islands sub-region and outside the Southern Ocean. Legend: E: East Antarctic sub-region; W: West Antarctic sub-region; G: South Georgia district; S: Subantarctic Islands subregion; M: Magellan sub-region; A: South America (north to M); O: outside Southern Ocean and South America; XX: new records for the Magellan sub-region. New material listed in bold face.

\section{GAMMARIDEA}

Fam. Acanthonotozomellidae

Acanthonotozomella barnardi Watling \& Holman, 1980

Acanthonotozomoides sublitoralis Schellenberg, 1931

Fam. Ampeliscidae

Ampelisca anversensis Karaman, 1975

Ampelisca composita Schellenberg, 1931

Ampelisca dentifera Schellenberg, 1931

Ampelisca gracilicauda Schellenberg, 1931

Ampelisca macrodonta Goeke, 1987

Ampelisca statenensis K.H. Barnard, 1932

gen. spp.

Fam. Amphilochidae

Amphilochus marionis Stebbing, 1888

Gitanopsis squamosa (Thomson, 1880)

Fam. Ampithoidae

Peramphithoe femorata (Kroyer, 1845)

W

G

A

W

$\mathrm{S}$
$\mathrm{S}$

$\mathrm{T}$

M

$\mathrm{O}$ ?

$\mathrm{O}$

Fam. Cheidae

Cheus annae Thurston, 1982

Fam. Colomastigidae

Colomastix castellata K.H. Barnard, 1932

Colomastix fissilingua Schellenberg, 1926

Fam. Corophiidae s.1.

Aora anomala Schellenberg, 1926

Aora cf. anomala Schellenberg, 1926

Corophium bonellii Milne Edwards, 1830

Corophium cylindricum (Say, 1818)

Gammaropsis (Gammaropsis) ctenura (Schellenberg, 1931)

Gammaropsis (Gammaropsis) cf. ctenura (Schellenberg, 1931)

Gammaropsis (Gammaropsis) deseadensis Alonso, 1981

Gammaropsis (Gammaropsis) longicornis Walker, 1906

Gammaropsis (Gammaropsis) longitarsus (Schellenberg, 1931)

Gammaropsis (Gammaropsis) monodi (Schellenberg, 1931)

Gammaropsis (Gammaropsis) remipes (K.H. Barnard, 1932)

Gammaropsis (Paranaenia) dentifera (Haswell, 1879)

Gammaropsis (Paranaenia) typica (Chilton, 1884)

Haplocheira balssi Schellenberg, 1931

Haplocheira barbimana robusta K.H. Barnard, 1932

Lembos argentinensis Alonso, 1992

?Lembos fuegiensis (Dana, 1853)

Fam. Cyproideidae

Victorhensenoides arntzi Rauschert, 1997

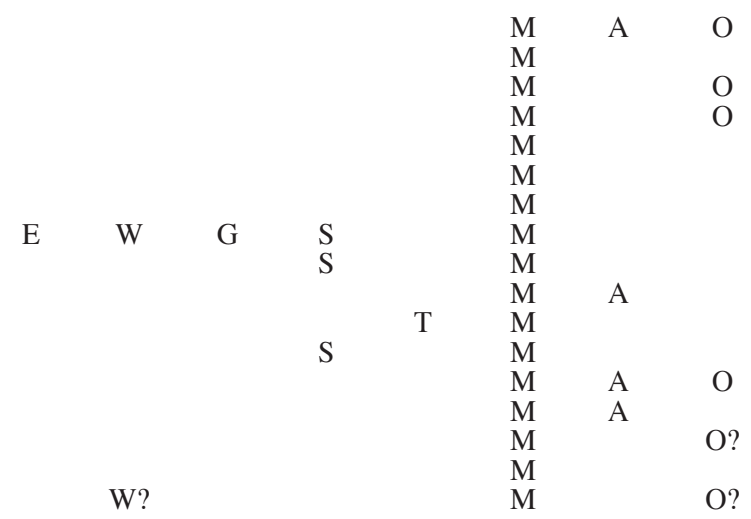

Fam. Dexaminidae

Atylus dentatus (Schellenberg, 1931)

Atylus villosus Bate, 1862

Atylus cf. villosus Bate, 1862

Atylus sp. Alonso, 1980

Paradexamine nana Stebbing, 1914

Paradexamine pacifica (Thomson, 1879)

Polycheria acanthocephala Schellenberg, 1931

Polycheria macrophtalma Schellenberg, 1931

Polycheria similis Schellenberg, 1931

Fam. Eophliantidae

Bircenna fulva Chilton, 1884

XX

Fam. Epimeriidae

Epimeria inermis Walker, 1903

Metepimeria acanthura Schellenberg, 1931

E W

A

O

$\mathrm{O}$

$\mathrm{O} ?$

$\mathrm{O}$ ?

Fam. Eusiridae s.1.

Atyloella dentata K.H. Barnard, 1932

Atyloella magellanica (Stebbing, 1888)
$\mathrm{O}$

A

A

A

M

M

M

M
A 
Table 1. (Cont.)

Eusiroides monoculoides (Haswell, 1879)

Eusirus antarcticus Thomson, 1880

Oradarea megalops (Nicholls, 1938)

Oradarea $c f$. megalops

Oradarea sp.n.I

Oradarea sp.n.2

Paramoera brachyura Schellenberg, 1931

Paramoera fissicauda (Dana, 1852)

Paramoera gregaria (Pfeffer, 1888)

Paramoera hermitensis K.H. Barnard, 1932

Paramoera obliquimana K.H. Barnard, 1932

Paramoera parva Ruffo, 1949

?Paramoera pfefferi Schellenberg, 1931

Paramoera sp. Stebbing, 1914

Paramoera sp. Monod, 1926

Paramoera sp. Ruffo, 1947

Rhachotropis antarctica K.H. Barnard, 1932

Rhachotropis schellenbergi Andres, 1982

Rhachotropis sp.n.

Tylosapis dentatus (Stebbing, 1888)

Tylosapis sp. $n$.

gen. spp.

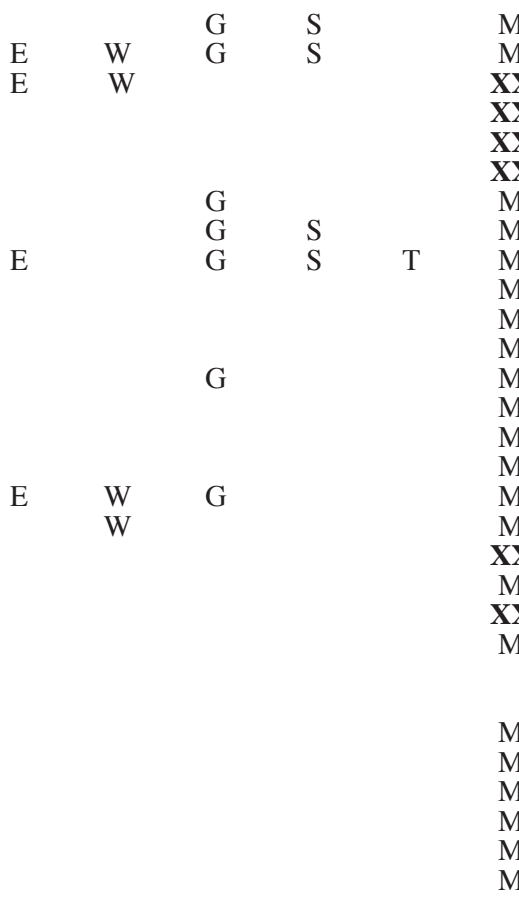

Fam. Exoedicerotidae

Bathyporeiapus magellanicus Schellenberg, 1931

Exoediceropsis affinis Alonso de Pina, 1997

Exoediceropsis lobata Alonso de Pina, 1997

Exoediceropsis chiltoni Schellenberg, 1931

Exoediceropsis cf. chiltoni Schellenberg, 1931

Metoediceros fuegiensis Schellenberg, 1931

Fam. Gammarellidae

Austroregia batei (Cunningham, 1871)

Austroregia huxleyana (Bate, 1862)

Austroregia regis (Stebbing, 1914)

Chosroes incisus Stebbing, 1888

Gondogeneia antarctica (Chevreux, 1906)

Gondogeneia dentata Alonso, 1986

Gondogeneia gracilicauda (Schellenberg, 1931)

Gondogeneia macrodon (Schellenberg, 1931)

Gondogeneia cf. macrodon (Schellenberg, 1931)

Gondogeneia patagonica Alonso, 1986

Gondogeneia simplex (Dana, 1852)

Gondogeneia thurstoni Alonso, 1989

Gondogeneia ushuaiae (Schellenberg, 1931)

Gondogeneia sp. (Ruffo, 1949)

Gondogeneia sp. 1

Gondogeneia sp. 2

Gondogeneia sp.n.

gen. sp.n.

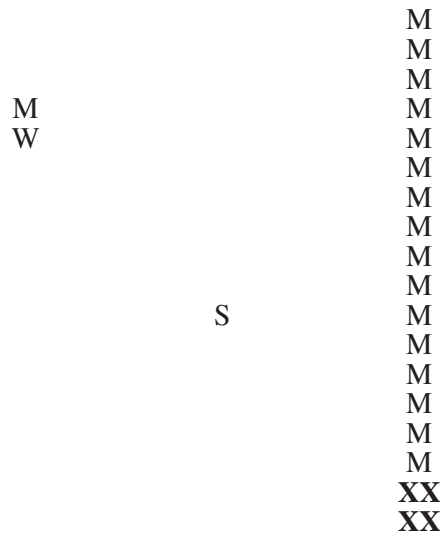

Fam. Gammaridae s.l. (Gammarida : Ceradocus group)

Maera eugeniae Schellenberg, 1931

M

Fam. Hyalidae

Hyale hirtipalma (Dana, 1852)

Hyale media (Dana, 1853)

Fam. Iphimediidae

Iphimedia imparilabia Watling \& Holman, 1980

Iphimedia macrocystidis (K.H. Barnard, 1932)

Iphimedia magellanica Watling \& Holman, 1980

Iphimedia multidentata (Schellenberg, 1931)

Labriphimedia vespuccii K.H. Barnard, 1931

Pariphimedia normani (Cunningham, 1871

Pseudiphimediella glabra (Schellenberg, 1931)

Pseudiphimediella cf. glabra (Schellenberg, 1931)

Pseudiphimediella nodosa (Dana, 1852)

Fam. Ischyroceridae

Cerapus sp. Alonso, 1980

Ischyrocerus hortator J.L. Barnard, 1964

Ischyrocerus sp. Alonso, 1986

Jassa alonsoae Conlan, 1990

Jassa justi Conlan, 1990 
Table 1. (Cont.)

Jassa marmorata Holmes, 1903

Jassa sp.

Pseudischyrocerus denticauda Schellenberg, 1931

Ventojassa georgiana (Schellenberg, 1931)

E

Fam. Laphystiopsidae

Prolaphystiopsis platyceras Schellenberg, 1931

W

G

S

$\mathrm{M}$

Fam. Leucothoidae

Leucothoe spinicarpa (Abildgaard, 1789)

$\begin{array}{lllll}\text { E } & \text { W } & \text { G } & \text { S } & \text { M }\end{array}$

$\mathrm{O}$

Fam. Liljeborgiidae

Liljeborgia falklandica K.H. Barnard, 1932

Liljeborgia longicornis (Schellenberg, 1931)

Liljeborgia macrodon Schellenberg, 1931

Liljeborgia cf. macrodon Schellenberg, 1931

Liljeborgia cf.octodentata Schellenberg, 1931

Liljeborgia quadridentata Schellenberg, 1931

Liljeborgia quinquedentata Schellenberg, 1931

Liljeborgia sp.n. 1

Liljeborgia sp.n. 2

Fam. Lysianassidae s.1.

Acontiostoma marionis Stebbing, 1888

Amaryllis sp. (or spp.) cf macrophtalma Haswell, 1879

Amaryllis sp.

Aristias antarcticus Walker, 1906

Aruga falklandica (K.H. Barnard, 1932

Erikus dahli Lowry \& Stoddart, 1987

Falklandia reducta (Schellenberg, 1931)

W

G

S

M

Lepidepecreoides sp.

Lysianopsis subantarctica (Schellenberg, 1931)

Orchomenella (Orchomenopsis) cavimana (Stebbing, 1888)

Orchomenella (Orchomenopsis) cavimana rostrata (Schellenberg, 1931)

Orchomenella (Orchomenopsis) chilensis (Heller, 1865)

Orchomenella (Orchomenopsis) sp.1

Orchomenella (Orchomenopsis) sp. $n$.

Orchomenella (Orchomenyx) schellenbergi (Thurston, 1972)

Pachychelium barnardi Alonso, 1993

Pachychelium cf. schellenbergi Lowry, 1984

Paralysianopsis odhneri Schellenberg, 1931

Parawaldeckia kidderi (Smith, 1876)

Pseudokoroga barnardi Schellenberg, 1931

Socarnoides unidentatus (Schellenberg, 1931)

Stephensenia haematopus Schellenberg, 1928

Stomacontion pepinii (Stebbing, 1888)

Stomacontion sp. 1

Stomacontion sp. 2

Tryphosella bispinosa (Schellenberg, 1931)

Tryphosella castellata (K.H. Barnard, 1932)

?Tryphosella paramoi (Schellenberg, 1931)

Tryphosella schellenbergi (Schellenberg, 1931)

?Tryphosella serrata (Schellenberg, 1931)

Tryphosoides falcatus Schellenberg, 1931

Uristes gigas Dana, 1849

Uristes serratus Schellenberg, 1931

Uristes subchelatus (Schellenberg, 1931)

Stenia magellanica Dana, 1852

gen. spp.

Fam. Melphidippidae

Melphidippa sp.

$\begin{array}{llll}\text { E } & \text { W } & \text { G } & \text { S } \\ \text { E } & & & \end{array}$

$M$

M
$M$

Fam. Ochlesidae

Curidia magellanica Coleman \& Barnard, 1991

W?

Fam. Oedicerotidae

Monoculodes sp.

Monoculopsis vallentini Stebbing, 1914

Oediceroides cinderella Stebbing, 1888

Oediceroides lahillei lahillei Chevreux, 1911

Oediceroides cf. macrodactylus Schellenberg, 1931

Oediceroides cf. newnesi (Walker, 1903)

Paraperioculodes brevirostris (Schellenberg, 1931) 
Table 1. (Cont.)

Paraperioculodes cystiferus (Schellenberg, 1931)

Paraperioculodes cf. cystiferus (Schellenberg, 1931)

gen. spp.

Fam. Pagetinidae

Pagetina reducta Holman \& Watling, 1981

Fam. Pardaliscidae

Pardalisca magellanica Schellenberg 1931

Pardalisca cf. magellanica Schellenberg, 1931

M

Fam. Phoxocephalopsidae

Eophoxocephalopsis rhachianensis Thurston, 1989

Phoxocephalopsis gallardoi Barnard \& Clark, 1984

Phoxocephalopsis cf. gallardoi Barnard \& Clark,1984

Phoxocephalopsis zimmeri Schellenberg, 1931

Puelche orensanzi Barnard \& Clark, 1982

Fam. Phoxocephalidae

Birubius ?rostratus (Dana, 1853)

Fuegiphoxus abjectus Barnard \& Barnard, 1980

Fuegiphoxus fuegiensis (Schellenberg, 1931)

Heterophoxus videns K.H. Barnard, 1930

Heterophoxus cf. videns K.H. Barnard, 1930

Metharpinia longirostris Schellenberg, 1931

Microphoxus cornutus (Schellenberg, 1931)

Phoxorgia sinuata (K.H. Barnard, 1932)

Phoxorgia cf. sinuata (K.H. Barnard, 1932)

Proharpinia antipoda Schellenberg, 1931

Proharpinia stephenseni (Schellenberg, 1931)

Proharpinia cf. stephenseni (Schellenberg, 1931)

Pseudfoxiphalus setosus Andres, 1991

Pseudharpinia dentata Schellenberg, 1931

Pseudharpinia obtusifrons (Stebbing, 1888)

gen. sp. 1

gen. sp. 2

gen. sp. 3

gen. sp. 4

Fam. Platyischnopidae

Eudevenopus gracilipes (Schellenberg, 1931)

Platyischnopus sp.

Fam. Pleustidae

Parepimeria irregularis (Schellenberg, 1931)

Fam. Podoceridae

Podocerus brasiliensis (Dana, 1853)

Podocerus cristatus rotundatus Schellenberg, 1931

Fam. Sebidae

Seba saundersii Stebbing, 1875

Seba subantarctica Schellenberg, 1931

Seba typica (Chilton, 1884 )

Seba sp. Homan \& Watling, 1983

Fam. Stegocephalidae

Andaniotes corpulentus (Thomson, 1882)

Andaniotes linearis K.H. Barnard, 1932

gen. sp. 1

gen. $s p .2$

gen.(nov.?) sp. n.1

gen.(nov.?) sp. n.2

W

W
W

G

$\mathrm{O}$ ?

A

A

A

G

A

A

S

Fam. Stenothoidae

Mesoproboloides cornutus (Schellenberg, 1926)

Metopoides cf. clavatus Schellenberg, 1931

Metopoides cf. heterostylis Schellenberg, 1926

Metopoides longicornis Schellenberg, 1931

Metopoides magellanicus (Stebbing,1888)

Metopoides sp.

Metopoides sp.n. 1

Metopoides sp.n. 2

Metopoides sp.n. 3

Probolisca elliptica (Schellenberg, 1931)

Probolisca cf. elliptica (Schellenberg, 1931)

$\mathrm{S}$ 
Table 1. (Cont.)

Probolisca nasutigenes (Stebbing, 1888)

Probolisca ovata (Stebbing, 1888)

Prometopa sp.n.

Pseudothaumatelson patagonicum Schellenberg, 1931

Scaphodactylus sp.n.

Stenothoe falklandica Schellenberg, 1931

Stenothoe magellanica Rauschert, 1997

Stenothoe cf. magellanica Rauschert, 1997

Thaumatelson herdmani Walker, 1906

Torometopa cf. andresi (Rauschert, 1990)

Torometopa compacta (Stebbing, 1888)

Torometopa crassicornis Schellenberg,1931

Torometopa crenatipalmata (Stebbing, 1888)

Torometopa cf. crenatipalmata (Stebbing, 1888)

Torometopa parallelocheir (Stebbing,1888)

Torometopa porcellana (K.H. Barnard, 1932)

Torometopa sp.n. 1

Torometopa sp.n. 2

Torometopa sp.n. 3

Torometopa sp.n. 4

Torometopa sp.n. 5

Torometopa sp.n. 6

Torometopa sp.n. 7

Torometopa sp.n. 8

Torometopa sp.n. 9

Fam. Stenothoidae, cont'd.

gen. spp.n.

E $\quad$ W

G

S
S

A

M A

XX

M

XX

XX

XX

$\mathrm{M}$

M

M

G

G $\quad \mathrm{S} \quad \mathrm{T}$

G

M

XX

XX

$\mathbf{X X}$

XX

XX

$\mathbf{X X}$

XX

XX

XX

M

Fam. Stilipedidae

gen. sp.

M

Fam. Synopiidae

Synopia sp.

Fam. Talitridae

Orchestia gammarellus (Pallas, 1776)

Orchestia scutigerula Dana, 1852

Orchestoidea tuberculata Nicolet, 1849

Protorchestia nitida (Dana, 1852)

Transorchestia chiliensis (Milne-Edwards, 1840)

Fam. Urohaustoriidae

Huarpe escofeti Barnard \& Clark, 1982

Fam. Urothoidae

Urothoe falcata Schellenberg, 1931

Fam. Zobrachoidae

Chono angustiarum Clark \& Barnard, 1987

Tonocote introflexidus Clark \& Barnard, 1988

Tonocote magellani Clark \& Barnard, 1986

\section{CAPRELLIDEA}

Fam. Phtisicidae

Aeginoides gaussi Schellenberg, 1926

Caprellina longicollis (Nicolet, 1849)

Dodecasella georgiana (Schellenberg, 1931)

Pseudoprotomima hedgpethi McCain \& Gray, 1971

Fam. Caprellinoididae

Caprellinoides mayeri (Pfeffer, 1888)

Dodecas elongata Stebbing, 1883

W

G

S
S

$\mathrm{M}$

$\mathrm{M}$

$\mathrm{M}$

M? A

$\mathrm{O}$

A

Fam. Caprellidae

Caprella equilibra Say, 1818

Caprella penantis Leach, 1814

Caprella ungulina Mayer, 1903

Caprella sp. McCain \& Gray, 1971

Fam. Pariambidae

Luconacia vemae McCain \& Gray, 1971

Triantella solitaria Mayer, 1903

Fam. Protellidae

Mayerella magellanica McCain \& Gray, 1971

Protella trilobata McCain \& Gray, 1971
$\mathrm{O}$ 
TABLE 2. - Taxonomic diversity of the benthic gammaridean amphipods of the Southern Ocean (updated from De Broyer and Jazdzewski, 1996, for the Magellan region; provisional identifications excluded)

\begin{tabular}{|c|c|c|c|c|c|c|c|}
\hline & $\begin{array}{c}\mathrm{N} \mathrm{spp} \\
(\text { N endemics) }\end{array}$ & $\begin{array}{c}\mathrm{N} \text { gen } \\
(N \text { endemics })\end{array}$ & $\begin{array}{c}\mathrm{N} \text { fam } \\
(\text { N endemics })\end{array}$ & & $\begin{array}{c}\mathrm{N} \mathrm{spp} \\
\text { (N endemics) }\end{array}$ & $\begin{array}{c}\mathrm{N} \text { gen } \\
(N \text { endemics })\end{array}$ & $\begin{array}{c}\mathrm{N} \text { fam } \\
(\text { N endemics) }\end{array}$ \\
\hline Magellan sub-region (this paper) & $\begin{array}{l}206 \\
(113)\end{array}$ & 113 & 42 & West Antarctic sub-region & $\begin{array}{c}376 \\
(197)\end{array}$ & 139 & 38 \\
\hline De Broyer and Jazdzewski (1996) & $\begin{array}{l}170 \\
(85)\end{array}$ & 104 & 38 & East Antarctic sub-region & $222(83)$ & 99 & 30 \\
\hline Subantarctic Islands sub-region & $\begin{array}{l}186 \\
(73)\end{array}$ & 111 & 38 & Total Antarctic region & $\begin{array}{c}470 \\
(369)\end{array}$ & $\begin{array}{l}175 \\
(47)\end{array}$ & 42 \\
\hline Total Subantarctic region & $\begin{array}{c}361 \\
(202)\end{array}$ & $\begin{array}{l}166 \\
(23)\end{array}$ & 47 & Total Southern Ocean & $\begin{array}{c}720 \\
(624)\end{array}$ & $\begin{array}{c}262 \\
(101)\end{array}$ & $\begin{array}{l}54 \\
(3)\end{array}$ \\
\hline
\end{tabular}

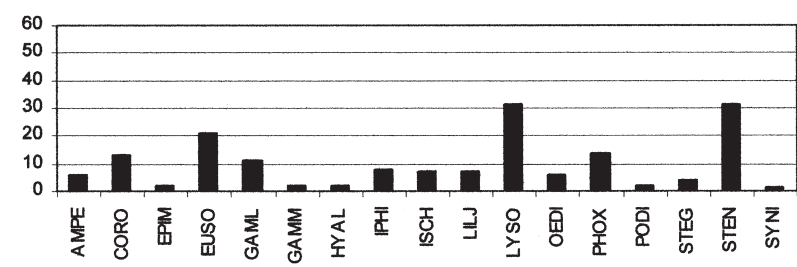

$$
\begin{gathered}
\mathbf{M} \\
\mathrm{n}=163 \\
\mathrm{n} \text { total }=206
\end{gathered}
$$

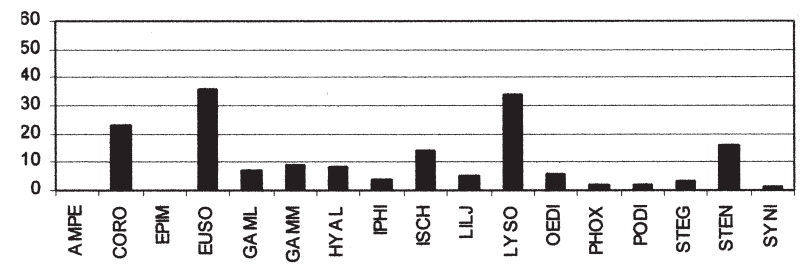

$$
\begin{gathered}
\mathrm{S} \\
\mathrm{n}=170 \\
\mathrm{n} \text { total }=196
\end{gathered}
$$

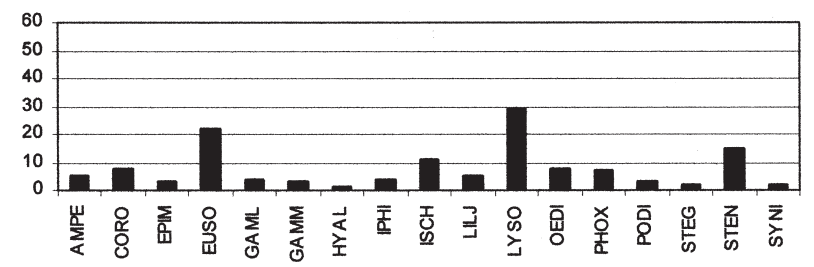

$$
\begin{gathered}
\mathbf{G} \\
\mathrm{n}=132 \\
\mathrm{n} \text { total }=153
\end{gathered}
$$

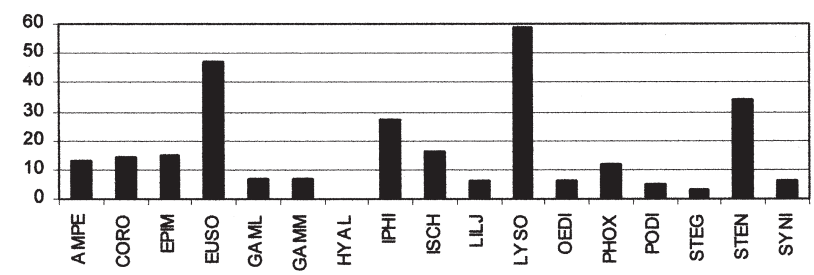

$$
\begin{gathered}
\mathbf{W} \text { (incl. G) } \\
\mathrm{n}=277 \\
\mathrm{n} \text { total }=323
\end{gathered}
$$

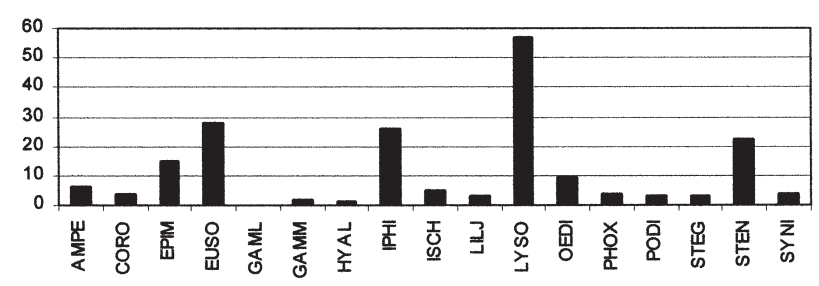

$$
\begin{gathered}
\mathbf{E} \\
\mathrm{n}=192 \\
\mathrm{n} \text { total }=216
\end{gathered}
$$

FIG. 1. - Comparative species richness of the 17 most speciose families of the different zoogeographic sub-regions of the Southern Ocean ( $\mathrm{n}$ $=$ cumulated number of species of these 17 families; $n$ total $=$ number of species of all families from the whole faunule; $n . b .: \mathrm{W}$ includes $\mathrm{G}$ in the calculation). M, A, S, G, W, E: see Table 1. AMPE: Ampeliscidae; CORO: Corophiidae s.1.; EPIM: Epimeriidae; EUSO: Eusiridae s.l.; GAML: Gammarellidae; GAMM: Gammaridae s.l.; HYAL: Hyalidae; IPHI: Iphimediidae; ISCH: Ischyroceridae; LILJ: Liljeborgiidae; LYSO: Lysianassidae s.l.; OEDI: Oedicerotidae; PHOX: Phoxocephalidae; PODI: Podoceridae; STEG: Stegocephalidae; STEN:

Stenothoidae; SYNI: Synopiidae. 
pus, Prometopa, Scaphodactylus, Synopia, Victorhensenoides, and a new genus of Gammaridae s.l.) is recorded for the first time or confirmed in case of previous doubtful records (Eusiroides and Platyischnopus).

The species richness of the 17 most speciose gammaridean families in the different zoogeograph- ical sub-regions of the Southern Ocean is compared in Figure 1.

With the discovery of the new species Victorhensenoides arntzi Rauschert, the family Cyproideidae was represented for the first time in the Magellan area by its collection during the "Victor Hensen" cruise 1994 (Rauschert, 1996). The

TABLE 3. - Scavenger amphipods and other organisms collected in baited traps in the area of the eastern entrance of the Beagle Channel (Joint Magellan "Victor Hensen" Campaign 1994)

\begin{tabular}{|c|c|c|c|c|c|c|c|}
\hline Station & Locality & Depth & $\begin{array}{l}\text { Hours } \\
\text { botton }\end{array}$ & Species & N. ind. & & \\
\hline 1147 & Isla Picton & $115 \mathrm{~m}$ & $72 \mathrm{~h}$ & $\begin{array}{l} \\
\text { ISOPODA } \\
\text { COPEPODA } \\
\text { DECAPODA }\end{array}$ & $\begin{array}{l}\text { Lysiana } \\
\text { Orchom } \\
\text { Orchom } \\
\text { Orchom } \\
\text { Tryphos } \\
\text { Tryphos } \\
\text { Cirolan } \\
\text { gen. sp. } \\
\text { Munida }\end{array}$ & $\begin{array}{l}\text { ae s.l.: } \\
\text { la (Orchomenopsis) cavimanus } \\
\text { la (Orchomenopsis) chilensis } \\
\text { la (Orchomenopsis) n.sp.1 } \\
\text { schellenbergi } \\
\text { chevreuxi } \\
\text { gen. sp.1 } \\
\text { rugosa (juv.) }\end{array}$ & $\begin{array}{r}30 \\
4792 \\
69 \\
51 \\
1298 \\
10 \\
10 \\
2\end{array}$ \\
\hline 1171 & Isla Picton & $40 \mathrm{~m}$ & $52 \mathrm{~h}$ & $\begin{array}{l}\text { AMPHIPODA } \\
\\
\text { ISOPODA } \\
\text { LEPTOSTRACA } \\
\text { DECAPODA }\end{array}$ & $\begin{array}{l}\text { Lysiana } \\
\text { Orchom } \\
\text { Tryphos } \\
\text { Tryphos } \\
\text { Cirolan } \\
\text { gen. sp. } \\
\text { Munida }\end{array}$ & $\begin{array}{l}\text { ae } \text { s.l.: } \\
\text { la (Orchomenopsis) chilensis } \\
\text { schellenbergi } \\
\text { chevreuxi } \\
\text { gen.sp.1 } \\
\text { rugosa (juv.) }\end{array}$ & $\begin{array}{r}9 \\
177 \\
29 \\
9 \\
1 \\
2\end{array}$ \\
\hline 1198 & Isla Picton & $60 \mathrm{~m}$ & $37 \mathrm{~h}$ & $\begin{array}{l}\text { ISOPODA } \\
\text { COPEPODA } \\
\text { PYCNOGONIDA }\end{array}$ & $\begin{array}{l}\text { Lysiana } \\
\text { Erikus } \\
\text { Orchom } \\
\text { Orchom } \\
\text { Tryphos } \\
\text { Ischyro } \\
\text { Eusirid. } \\
\text { Oedicer } \\
\text { Stenoth } \\
\text { Stegoce } \\
\text { Cirolan } \\
\text { Sphaero } \\
\text { gen. sp. } \\
\text { gen. sp. } \\
\text { gen. sp. }\end{array}$ & $\begin{array}{l}\text { ae s.l.: } \\
\text { la (Orchomenopsis) cavimanus } \\
\text { la (Orchomenopsis) chilensis } \\
\text { schellenbergi } \\
\text { lae gen. sp.1 } \\
\text { l. Paramoera sp.1 } \\
\text { ae gen. sp. } \\
\text { e gen. sp. } \\
\text { idae gen. sp. } \\
\text { gen. sp.1 } \\
\text { idae gen. sp. }\end{array}$ & $\begin{array}{r}5 \\
1 \\
1 \\
25 \\
2 \\
1 \\
3 \\
1 \\
1 \\
11 \\
2 \\
1 \\
6 \\
1\end{array}$ \\
\hline 1226 & Isla Lennox & $\mathrm{x} 24 \mathrm{~m}$ & $49 \mathrm{~h}$ & $\begin{array}{l}\text { AMPHIPODA } \\
\text { ISOPODA } \\
\text { MYSIDACEA } \\
\text { POLYCHAETA } \\
\text { AGNATHA }\end{array}$ & $\begin{array}{l}\text { Lysiana } \\
\text { Trypho. } \\
\text { Ischyro } \\
\text { Cirolan } \\
\text { gen. sp. } \\
\text { gen. sp. } \\
\text { Myxini }\end{array}$ & $\begin{array}{l}\text { ae s.l.: } \\
\text { schellenbergi } \\
\text { lae gen. sp. } 2 \\
\text { gen. sp.1 } \\
\text { gen. sp. }\end{array}$ & $\begin{array}{r}7 \\
1 \\
12 \\
1 \\
2 \\
68\end{array}$ \\
\hline 1230 & Punta Aaron & $\mathrm{n} 50 \mathrm{~m}$ & $11 \mathrm{~h}$ & $\begin{array}{l}\text { AMPHIPODA } \\
\text { ISOPODA } \\
\text { DECAPODA } \\
\text { AGNATHA }\end{array}$ & $\begin{array}{l}\text { Lysiana } \\
\text { Cirolan } \\
\text { Munida } \\
\text { Myxini }\end{array}$ & $\begin{array}{l}\text { ae gen. sp. } \\
\text { gen. sp.1 } \\
\text { rugosa (juv.) } \\
\text { en. sp. }\end{array}$ & $\begin{array}{r}2 \\
3 \\
2 \\
12\end{array}$ \\
\hline Total & 5 sta. & & $221 \mathrm{~h}$ & $\begin{array}{l}\text { AMPHIPODA } \\
\text { ISOPODA } \\
\text { COPEPODA } \\
\text { LEPTOSTRACA } \\
\text { DECAPODA } \\
\text { PYCNOGONIDA } \\
\text { POLYCHAETA } \\
\text { AGNATHA }\end{array}$ & $\begin{array}{l}\text { gen: } 10 \\
\text { gen: } 2 \\
\text { gen: } 2 \\
\text { gen: } 1 \\
\text { gen: } 1 \\
\text { gen: } 1 \\
\text { gen: } 1 \\
\text { gen: } 1\end{array}$ & $\begin{array}{l}\text { spp: } 12 \\
\text { spp: } 2 \\
\text { spp: } ? \\
\text { spp: } 1 \\
\text { spp: } 1 \\
\text { spp: } 1 \\
\text { spp: } 1 \\
\text { spp: } 1\end{array}$ & $\begin{array}{r}6505 \\
45 \\
11 \\
1 \\
6 \\
1 \\
2 \\
80\end{array}$ \\
\hline
\end{tabular}


family was previously unknown from the Subantarctic and Antarctic regions. Related genera are known from Australia, New Zealand and the Mediterranean Sea.

The family Stenothoidae, under revision by one of us (M.R.), received particular attention. Stenothoids are widespread in the Southern Ocean where they constitute the third most speciose gammaridean family. Representatives of this family are often overlooked or misidentified due to their small size. Their ecology remains poorly known. They could be associated with different sessile benthic organisms like algae, Hydrozoa, Ascidiacea, Porifera, or Octocorallia. Thirteen species were previously known from the Magellan area (De Broyer and Jazdzewski, 1993); 18 additional species were collected during the new campaigns. These new findings make the Stenothoidae the most speciose family for the Magellan area, along with the
Lysianassidae s.l. (Fig. 1). Until now, the genus Scaphodactylus Rauschert and Andres appeared to be endemic to the South Shetland Islands but its occurrence is extended now into the Magellan area where it was detected for the first time during the "Vidal Gormaz" cruise 1996 (Estrecho Nelson, $51^{\circ} 41,40^{\prime} \mathrm{S} 73^{\circ} 13,40^{\prime} \mathrm{W}, 90 \mathrm{~m}$ depth).

\section{Composition of the scavenger component of the amphipod fauna}

The bulk of the species collected by baited traps during the "Victor Hensen" campaign was made up of lysianassoids (Table 3). In addition to Amphipo$\mathrm{da}$, a number of Isopoda (Cirolanidae) and Agnatha (Myxinidae) and few (accidental?) Copepoda (2 spp, 6 ind.), Leptostraca (1 spp, 1 ind.), Decapoda (Munida subrugosa juv.), Pycnogonida, and Polychaeta were also caught in baited traps.

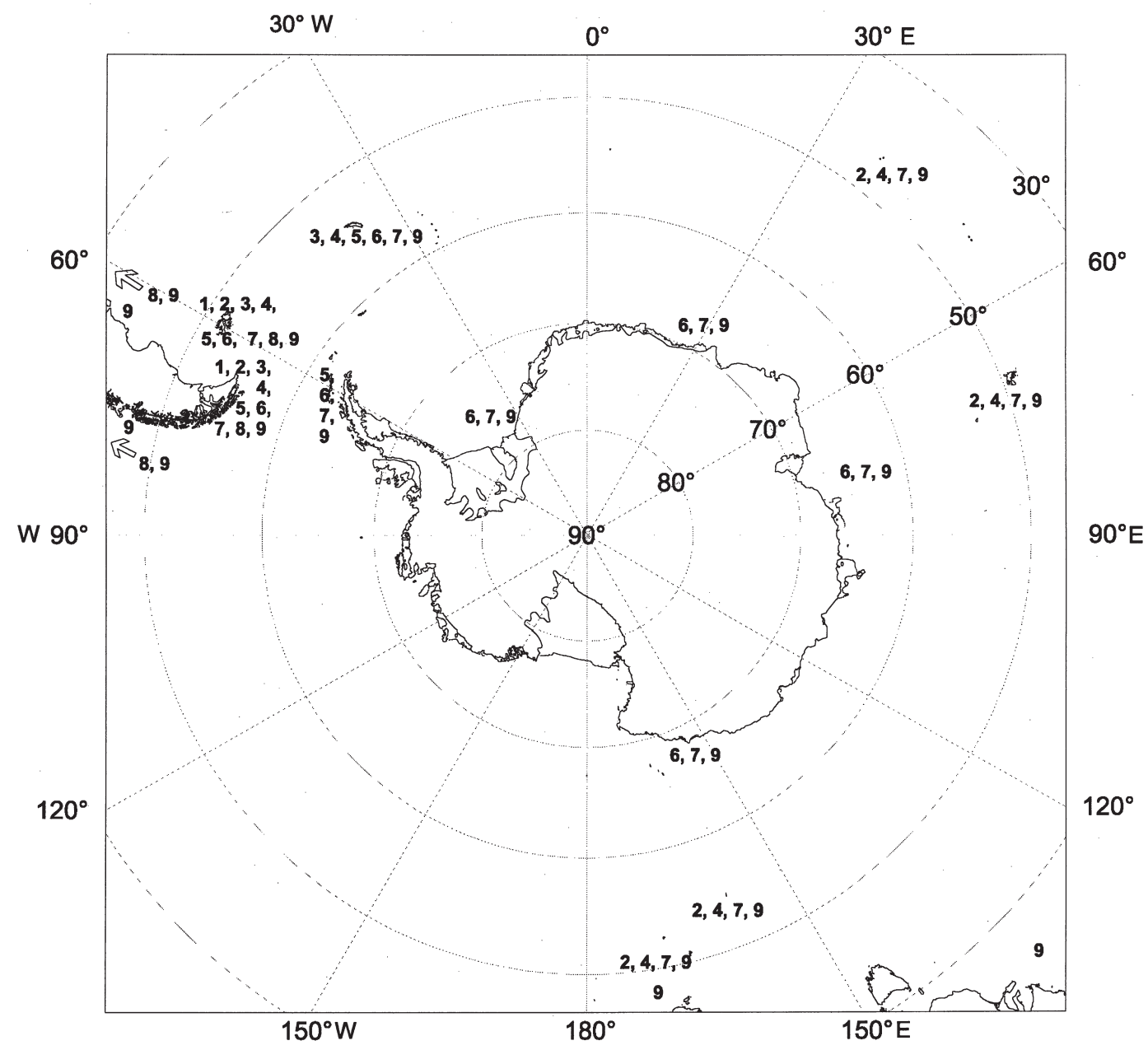

FIG. 2. - The different types of geographical distribution of the Magellan amphipod fauna (1. Endemic; 2. Circumsubantarctic; 3. Magellan subregion + South Georgia; 4. Magellan subregion + West Antarctic + Subantarctic Islands; 5. Magellan + West Antarctic; 6. Magellan + Antarctic; 7; Circumpolar; 8. South America; 9. Austral; 10. "Cosmopolitan"). For significance of numbers see text. 


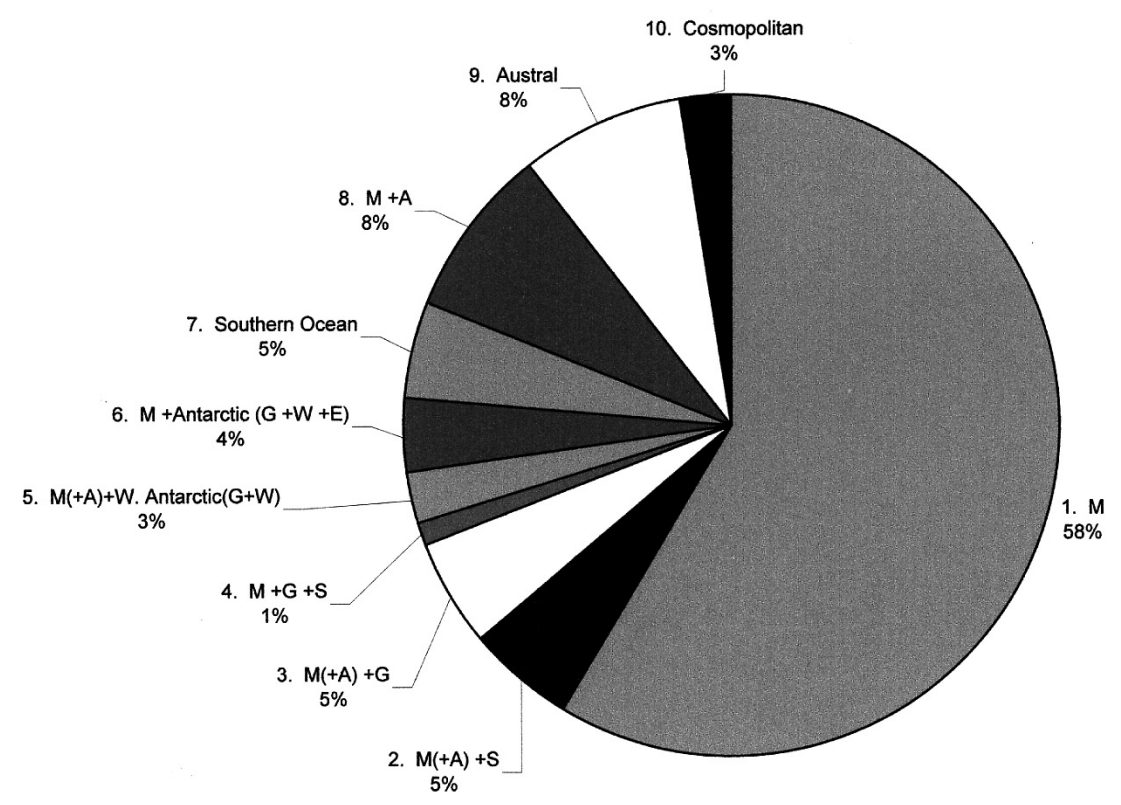

FIG. 3. - Zoogeographical affinities of the Magellan gammaridean amphipods. Legend: E: East Antarctic sub-region; W: West Antarctic subregion; G: South Georgia district; S: Subantarctic Islands sub-region; M: Magellan sub-region; A: South America (north to M); Austral: South America and Southern Hemisphere.

\section{Zoogeographical affinities of Magellan amphipod fauna}

Benthic gammaridean amphipods of the Magellan area exhibit different patterns of distribution in the Southern Ocean or the Southern Hemisphere (Fig. 2 and 3):

1. Endemic: Magellan sub-region $(\mathrm{M}=112 \mathrm{spp})$. Examples: Orchomenella (Orchomenopsis) chilensis, Torometopa crassicornis.

2. Circumsubantarctic: Magellan sub-region + Subantarctic Islands $(\mathrm{M}+\mathrm{S}=8 \mathrm{spp} ;+\mathrm{A}=2 \mathrm{spp})$, e.g. Acontiostoma marionis, Stomacontion pepinii, Gammaropsis longitarsus.

3. Magellan sub-region + South Georgia $(\mathrm{M}+\mathrm{G}=7$ $\mathrm{spp} ;+\mathrm{A}=3 \mathrm{spp}$ ), e.g. Metopoides magellanicus, Seba subantarctica.

4. Magellan sub-region + South Georgia + Subantarctic Islands $(\mathrm{M}+\mathrm{G}+\mathrm{S}=2 \mathrm{spp})$ : Orchomenella (Orchomenyx) schellenbergi, Torometopa parallelocheir.

5. Magellan sub-region + West Antarctic, including South Georgia $(\mathrm{M}+\mathrm{W}+\mathrm{G}=3 \mathrm{spp} ;+\mathrm{A}=2 \mathrm{spp})$, e.g. Gondogeneia antarctica, Oediceroides lahillei.

6. Magellan + Antarctic $(\mathrm{M}+\mathrm{G}+\mathrm{W}+\mathrm{E}=7 \mathrm{spp})$, e.g. Rhachotropis antarctica, Paralysianopsis odhneri.

7. Circumpolar (whole Southern Ocean; $\mathrm{M}+\mathrm{S}+\mathrm{G}+\mathrm{W}+\mathrm{E}=9 \mathrm{spp})$, e.g. Aristias antarcticus, Colomastix fissilingua, Paramoera gregaria.
8. South American: Magellan + north to Chile and Argentina (in some cases to Peru or Brasil); $\mathrm{M}+\mathrm{A}=$ $16 \mathrm{spp}$, e.g. Ampelisca gracilicauda, Peramphithoe femorata, Tryphosites chevreuxi.

9. Austral (= widely distributed in South America and the Southern Hemisphere); 15 spp: e.g. Gitanopsis squamosa, Hyale hirtipalma, Probolisca ovata.

10. "Cosmopolitan" (5 spp): species distributed worldwide (e.g. Leucothoe spinicarpa) or occurring in the Northern Hemisphere (e.g. Corophium bonellii) or circumtropical (e.g. Podocerus brasiliensis).

\section{DISCUSSION}

\section{Taxonomical and zoogeographical preliminary results}

In addition to an important catch of unknown species and new records for the area, the abundance of material collected will allow revision of a great part of the Magellan fauna. This taxonomic work takes place in the framework of a general revision of the Southern Ocean amphipod fauna, undertaken by the "Antarctic Amphipodologists Network" (Andres, Bellan-Santini, Berge, Coleman, Conlan, De Broyer (coord.), Hendrycks, Jazdzewski, Rauschert, Takeuchi, Thurston). A brief comparison of the dif- 
ferent Southern Ocean faunules (Fig. 1) showed that the Magellan gammarideans are dominated by lysianassoids (which are however about half the number of Antarctic species), stenothoids and eusiroids. The high number of stenothoid species -as in the West Antarctic- is probably a result of the relative importance of the study effort. Gammarellids (which here comprise a significant part of species formerly attributed to Pontogeneiidae) appear the most diverse in the Magellan area. Thirty-three (16 $\%$ ) of the benthic species of the Magellan subregion co-occur in the Antarctic region (12 spp or $6 \%$ extend only to South Georgia): they belong mostly to the free-living Eusiridae (10 spp) and Lysiannassoidea (8 spp), to Stenothoidea living in association with different benthic animals or algae (8 spp), and to the burrowing Phoxocephalidae (4 spp).

The new material should also allow reevaluation of the zoogeographical affinities and tracing of the origin(s) and dispersal of the Magellan amphipod fauna in comparison with the traits of the West- and East Antarctic faunas. This preliminary zoogeographical analysis showed than an important part (not less than $29 \mathrm{spp}$ ) of the species occurring in the Magellan region are distributed also along the South American shelf, in the Atlantic ocean sometimes to Brazil (see for instance Wakabara et al., 1991) or to Peru on the Pacific side. The species with extended distribution, in particular the so-called "cosmopolitans" or the australasian species recorded in the southernmost part of South America, most often demand careful re-examination and confirmation of their identity.

\section{Scavenger fauna}

Trap samples usually provide scavenging species which are not commonly taken by trawls, sledge and dredge. They also give indication of the relative importance of the scavenger component in benthic communities. The most successful sample was taken at "Victor Hensen" station 1147 (72h at $115 \mathrm{~m})$ and is probably due to the longer residence time on the bottom. The transect off Isla Picton ("Victor Hensen" stations 1147, 1171, 1198) indicates the probable preference of Orchomenella chilensis and Tryphosites chevreuxi for depths below 100m and of Tryphosella schellenbergi for depths shallower than $60 \mathrm{~m}$. Except detailed analysis of population structure or stomach contents of the samples, more precise interpretation of trap results (e.g. selective attractivity to different baits) seems difficult due to the small number of operations, the different residence times on the bottom and the use of non standard baits. At station 1198, traps contained a small number of amphipods belonging to species or families not usually known as scavengers and their attraction to bait should be confirmed. Some samples from stations shallower than $50 \mathrm{~m}$ i.e. sta. 1226 $(49 \mathrm{~h}$ at $24 \mathrm{~m})$ and sta. $1230(11 \mathrm{~h}$ at $50 \mathrm{~m})$ were partly spoiled due to significant presence of Myxinidae in the traps which provided abundant mucus and could have ingested trapped amphipods.

\section{ACKNOWLEDGEMENTS}

The authors are very grateful to masters and crews of the RV "Victor Hensen", "Polarstern" and "Vidal Gormaz" for their help and their efficiency during the different campaigns. W. Vader (Troms $\varnothing$ ), L. Watling (Walpole, Maine) and an anonymous referee made useful suggestions on the manuscript. C.D.B. expresses his sincere thanks to Prof. W. Arntz and the Alfred-Wegener-Institut for the invitation to participate in the Joint Magellan "Victor Hensen" Campaign 1994. The Leopold III Fund for Nature Exploration and Conservation provided travel funds. C.D.B. was partly supported by grant A4/DD/B02 within the "OSTC Belgian Antarctic Research Programme - Phase IV".

\section{REFERENCES}

Alonso de Pina, G.M. - 1997. New Exoediceropsis Schellenberg (Crustacea: Amphipoda: Exoedicerotidae) from the Argentine continental shelf and redescription of E. chiltoni Schellenberg. J. Nat. Hist., 31: 85-97.

Arntz, W.E. and M. Gorny. - 1996. Cruise report of the Joint Chilean-German-Italian Magellan "Victor Hensen” Campaign in 1994. Ber. Polarforsch., 190: 1-113.

Arntz, W.E., A. Buschmann, K.H. George, D. Gerdes, M. Gorny, M.A. Lardies, K. Linse, A. Montiel, E. Mutschke, M. Rauschert and C. Ríos. - 1997. Benthos investigations. Ber. Polarforsch., 239: 94-97, 123-124.

Brandt, A., K. Linse and U. Weber. - 1996. Abundance and diversity of peracarid taxa (Crustacea, Malacostraca) along a transect through the Beagle Channel, Patagonia. Polar Biol., 18: 83-90.

De Broyer, C. and K. Jazdzewski. - 1993. Contribution to the marine biodiversity inventory. A checklist of the Amphipoda (Crustacea) of the Southern Ocean. Doc. Trav. Inst. roy. Sci. nat. Belg., 73: 1-154.

De Broyer, C. and K. Jazdzewski. - 1996. Biodiversity of the Southern Ocean: towards a new synthesis for the Amphipoda (Crustacea). Boll. Mus. Civ. St. Nat. Verona, 20(1993): 547568.

De Broyer, C. and M. Rauschert. - 1996. Biodiversity and ecological roles of the amphipod crustaceans of the Antarctic and Magellan Region: a comparison. In: W. Arntz and M. Gorny (eds.), Cruise report of the Joint Chilean-German-Italian Magellan "Victor Hensen" Campaign in 1994. Ber. Polarforsch., 190: 55-57.

Fahrbach, E. and D. Gerdes. - 1997. Die Expedition ANTARKTIS 
XIII/4-5 des Forschungsschiffes "Polarstern" 1996. Ber. Polarforsch., 239: 1-126.

Hedgpeth, J.W. - 1969. Distribution of selected groups of marine invertebrates in waters south of $35^{\circ} \mathrm{S}$ latitude. Ant. Map Folio Ser., Amer. Geogr. Soc., New York, Folio, 11: 1-29.

Mutschke E., C. Ríos and M. Gorny. - 1995. Estudio bentónico en fiordos y canales patagónicos y fueginos. Informe de crucero de investigación científica marina a los fiordos y canales adyacentes a Campos de Hielo Sur. Univ. Magallanes, Punta Arenas: $72-85$.
Rauschert, M. - 1996. Erstnachweis der Familie Cyproideidae (Crustacea: Amphipoda: Gammaridea) in der MagellanRegion. Mitt. Zool. Mus. Berlin, 72: 199-206.

Rauschert, M. - 1998. Stenothoe magellanica sp. n. (Crustacea, Amphipoda, Gammaridea, Stenothoidae) aus dem Magellangebiet von Südchile 1. Mitt. Mus. Nat. kd. Berlin, Zool. Reihe, 74 (1): 43-48.

Wakabara, Y., A.S.Tararam, M.T. Valério-Berardo, W. Duleba and F. P. Pereira Leite. - 1991. Gammaridean and caprellidean fauna from Brazil. Hydrobiologia, 223: 69-77. 